\title{
Anti-proliferation of Melanoma Cells and Immune Stimulation by the Cyanobacterial Indole-alkaloid Scytonemin
}

Jadan Evans (evanjm05迎fw.edu)

Аагоп Jones (joneaa@ilu.edu)

Elliott Blumenthal (blumenth回pfw.edu)

*Tanya Soule (saulet国pfw.edu)

Department of Biology, Purdue University Fort Wayne

${ }^{*}$ Corresponding Author

Department of Biology, Purdue University Fort Wayne, 2101 E Coliseum Blvd., Fort Wayne, Indiana 46845

Manuscript received 29 January, 2021; accepted 15 June, 2021

Keywords: scytonemin, melanoma, spleen cells, anti-proliferative, natural products 


\section{Abstract}

Under the stress of ultraviolet radiation some cyanobacteria synthesize scytonemin, a protective pigment against DNA photodamage. In addition to photoprotection, scytonemin has been shown to have an anti-proliferative effect on various types of malignant cells. In this study the effect of scytonemin on melanoma and spleen cells was assessed both in vitro using tissue cultures and in vivo in mice models. Melanoma and spleen cells were exposed to 0.08 to $10 \mu \mathrm{M}$ of scytonemin, and cell proliferation was measured using tritiated thymidine uptake. The data suggest that scytonemin acts as an inhibitor for melanoma cells in a concentration-dependent manner while enhancing the proliferation of spleen cells, suggesting that it can potentially augment the immune response. Furthermore, mice injected with melanoma cells and scytonemin produced fewer tumors than mice that did not receive scytonemin, although the data were not significant. This study adds to the growing body of research that scytonemin may be beneficial as a future anticancer agent to prevent tumor cell growth. 


\section{Introduction}

According to the Centers for Disease Control, skin cancer is the most common type of cancer in the world, with 85,868 people in the United States diagnosed with melanomas of the skin in 2017 (3). Consequently, the demand for a product that is both effective in killing tumor cells and safe for an individual to take is as great as it has ever been. Since ultraviolet radiation (UVR) plays a major role in skin cancer, potential treatments could explore photoprotective effects of various compounds against solar UVR. Long- wavelength (UVA) in the range of $320-400 \mathrm{~nm}$, plays a role in longterm skin damage contributing to aging skin due to its deep penetration of the epidermis and dermis. UVA is known to damage keratinocytes, which are found in the basal layer of the epidermis where most skin cancers occur (8). Shortwavelength UVB in the range of $280-320 \mathrm{~nm}$ is the major contributor in sunburns and contributes to skin cancer alongside UVA by directly damaging DNA and proteins (17). All living cells, including bacteria, can be harmed by UVR (8). As photosynthetic bacteria regularly exposed to UVR, cyanobacteria have developed several mechanisms to defend themselves against its harmful effects. These include physical migration away from UVR (1), synthesis of UVshock proteins (4), up-regulation of antioxidant defenses (14), and down-regulation of UVR- sensitive proteins (7). Of particular interest is the ability of some cyanobacteria to synthesize scytonemin, a photoprotective sheath pigment that protects primarily against UVA radiation (5). Scytonemin is a lipophilic, yellow-brown, indole-alkaloid pigment (Fig.
1) (10) that efficiently absorbs UVA in vivo at $370 \mathrm{~nm}$ (6). It is produced by certain species of cyanobacteria where it is induced upon exposure to UVA, and is then deposited into the sheath surrounding the cells (5).

In addition to its photoprotective properties, scytonemin demonstrates anti- inflammatory and anti-proliferative qualities. Tissue hyperplasia is a hallmark feature of hyperproliferative and inflammatory pathologies, such as rheumatoid arthritis, psoriasis, asthma, and cancer (15). Scytonemin has been shown to exhibit anti-inflammatory properties that could potentially treat these diseases (11, $15,16)$. For example, the topical application of scytonemin on mouse ear edema reduced swelling compared to mice receiving no treatment (16). Furthermore, several studies argue that scytonemin inhibits cell proliferation through mechanisms of cell cycle arrest $(15,16,18,19)$. Scytonemin has also been shown to interrupt hyperproliferation of renal cancer cells (18) and slow the proliferation of multiple myeloma cells (19). Possibly the most thorough study on the anti-proliferative properties of scytonemin was that it could hinder actively proliferating cells, including malignant Jurkat $\mathrm{T}$ cells, rheumatoid synovial fibroblasts implicated in arthritis, human lung fibroblasts, and human umbilical vein endothelial cells. This study also demonstrated that scytonemin was not cytotoxic towards non-proliferative human monocytes (15). These results convey the possibility for scytonemin to halt malignant cell growth without harming other body cells, which is a quality particularly sought after in cancer treatment research.

\section{Figure 1.}

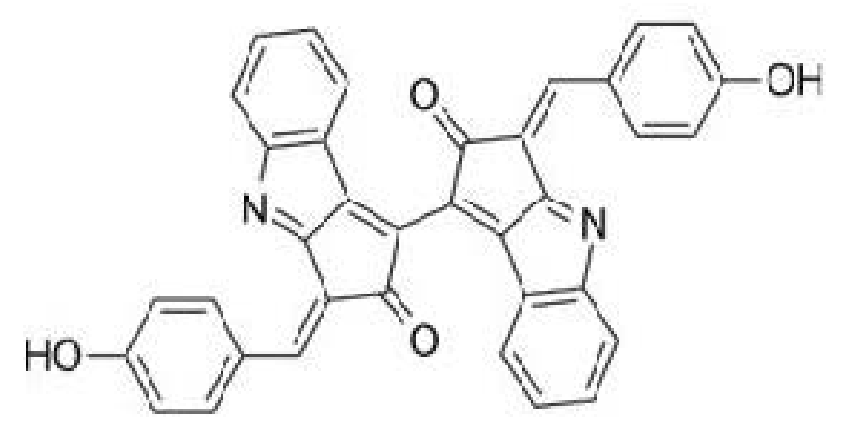

Figure 1. Structure of scytonemin (Proteau et. al. 1993).

56 | Fine Focus 
Since the literature suggests that the UV-protective agent scytonemin is implicated in cell cycle arrest, this study seeks to determine whether scytonemin will have anti-proliferative effects against melanoma cell growth in animals. Therefore, the objectives of this study were to examine both the in vitro melanoma and spleen cell proliferation in the presence of different concentrations of scytonemin, as well as the in vivo effect of scytonemin on tumor growth in mice. If scytonemin inhibits melanoma cell proliferation without equally inhibiting spleen cell proliferation, then it has potential as a future therapy for malignant cells and should be further explored as an anti-tumor therapy. However, if scytonemin affects melanoma and spleen cells alike, then it could potentially harm tissue and might not result in the production of an effective therapy. While the findings of other studies already indicate that scytonemin slows malignant proliferation $(15,16,18,19)$, this study takes a slightly different angle by comparing the effects of scytonemin on cancerous cell proliferation (i.e., melanomas) to healthy noncancerous cell growth (i.e., spleen cells) from other tissues in the same organism. Spleen cells were chosen over skin cells, for example, as the healthy non-cancerous control cells because they are more likely to interact with a therapeutic agent that enters the bloodstream. Furthermore, the spleen plays a critical role in activating immune cells in response to bloodborne antigens. Therefore, the hypothesis of this study is that in the presence of scytonemin, melanoma growth will decrease in tissues and animals while spleen cell proliferation will increase.

\section{Methods}

\section{Melanoma and Spleen Cell Assays}

B16-F1 melanoma cells were purchased from the American Type Culture Collection (ATCC(C CRL-6323) (2). Spleen cells were extracted from male C57BL/6 mice obtained from Charles River Labs that were 4-6 months old at the time of extraction. For each assay, a final concentration of $5 \times 104$ melanoma or 1 x 106 spleen cells were placed in each well of a 96-well plate and assayed in triplicate for each condition. Samples treated with scytonemin received $10 \mu \mathrm{L}$ HPLCpurified scytonemin (a gift from Benjamin Philmus, Oregon
State University) diluted in DMSO with final concentrations of 10 to $0.08 \mu \mathrm{M}$. To better determine the effect of scytonemin on spleen cell growth, the mitogen concanavalin A (Con A), which stimulates T lymphocyte proliferation, was added at a concentration of $0.1 \mu \mathrm{g}$ per well in a volume of $200 \mathrm{ul}$ of RPMI-1640 media plus 10\% FBS, to determine whether scytonemin inhibited rapidly proliferating spleen cells (12). Spleen cell plates were incubated for 48 hours, then $3 \mathrm{H}$-thymidine was added followed by a second incubation for 24 hours, all of which took place at $37^{\circ} \mathrm{C}$ under $10 \%$ $\mathrm{CO} 2$. A cell harvester was then used to transfer the cells onto filters, which were washed approximately ten times using phosphate buffered saline (PBS). Filters were then placed into counting tubes with EcoLume ${ }^{\mathrm{TM}}$ Scintillation Cocktail (MP Biomedicals, Solon, Ohio) to count the incorporated $\mathrm{H} 3$-thymidine using a Beckman scintillation counter. All assays were done in triplicate and controls received no scytonemin or DMSO treatment. Previous experiments in our lab have demonstrated that DMSO does not have an inhibitory effect on melanoma cell proliferation (unpublished data). Cell counts were averaged and compared to control groups to determine the percent proliferation relative to the control groups. All statistical tests were done using ANOVA and TukeyHSD post-hoc comparisons in R Studio v1.2.1335 (13).

\section{In Vivo Assays}

For in vivo scytonemin experiments against melanoma tumor cells, 18 male C57/BL6 mice were obtained from Charles River Labs that were approximately 14 weeks old at the time of experimentation. Each mouse was weighed before the initial intraperitoneal (IP) injection of scytonemin diluted in DMSO to a final concentration of $3.5 \mu \mathrm{Mg}$-1. Scytonemin was then administered at $3.5 \mu \mathrm{M}$ g-1 daily for two weeks through IP injections into nine treated mice, and the other nine mice received the same volume of sterile saline that had an equal concentration of DMSO as the scytonemintreated animals. After two weeks a single tail vein injection of melanoma cells was performed. Melanoma cells were diluted to $5 \times 106$ melanoma cells $\mathrm{ml}-1$ in sterile saline, and $0.1 \mathrm{ml}$ of this preparation was injected into the tail vein of all 18 mice. IP injections of scytonemin were continued for an additional week and the mice were monitored for any health changes Volume Seven | 57 
for two additional weeks while the tumors grew. After two weeks of no injections eight mice in each group survived and were weighed once more and sacrified. When injected into the tail vein of syngeneic mice, melanoma cells migrate to the lungs and produce dark colonies. Upon sacrifice the lungs were teased apart and the melanoma tumor cell colonies were counted on and within the lung tissue and the counts were compared against the control mice that did not receive scytonemin using an ANOVA with TukeyHSD post-hoc analysis. All animal experiments were performed according to IACUC Protocol \#1111000244.

\section{Results}

\section{Melanoma and Spleen Cell Assays}

The melanoma cell assays showed that in the presence of $10 \mu \mathrm{M}$ scytonemin, the percent inhibition compared to the control was highest at $87.60 \% \pm 1.86$ while in the presence of $2 \mu \mathrm{M}$ scytonemin, the inhibition decreased to $52.03 \% \pm$ 1.76 (Fig. 2). The percent inhibition compared to the control was significant only at $10 \mu \mathrm{M}$ and $2 \mu \mathrm{M}$ scytonemin ( $<<$ $0.0001)$. The percent inhibition generally increased as the concentration of scytonemin increased, indicating a positive relationship among these variables.

For the spleen cell assays, in all treatments except the 10 $\mu \mathrm{M}$ scytonemin dilution without Con A $(65.28 \% \pm 5.34)$, the percentage proliferation relative to the control was over $100 \%$ (Fig. 3). For the spleen cells receiving the mitogen Con $\mathrm{A}$, the percentage proliferation relative to the control was $211.14 \% \pm 53.24$ for the most dilute concentration of scytonemin, $0.08 \mu \mathrm{M}$, peaking at $387.00 \% \pm 79.34$ with 2 $\mu \mathrm{M}$ scytonemin. The percent stimulation compared to the control was significant only at $2 \mu \mathrm{M}$ scytonemin, with $\mathrm{p}=$ 0.0012 regardless of whether Con A was used. The results of the assays with and without Con A display the same general trends, suggesting that the presence of the mitogen was not a confounding variable. Scytonemin did not demonstrate any inhibition of these spleen tissue cultures while the $2 \mu \mathrm{M}$ and $10 \mu \mathrm{M}$ levels showed significant inhibition of melanoma cell growth.

58 | Fine Focus

\section{In Vivo Assays}

After treatment, there was no significant difference in the weights of untreated mice $(28.44 \pm 0.49 \mathrm{~g})$ and those treated with scytonemin $(29.63 \pm 0.48 \mathrm{~g})$. There were also no significant differences in the number of melanoma tumors in untreated mice $(205.5 \pm 51.29)$ versus those treated with scytonemin $(142.50 \pm 30.65)$.

\section{Discussion}

Mechanistically, scytonemin inhibits the hyperproliferation of cells by targeting multiple enzymes implicated primarily in cell cycle regulation. For instance, one study examined the effect on several kinases, including PLK1, CDK1/cyclin $\mathrm{B}$, checkpoint kinase 1, protein kinase $\mathrm{A}$, protein kinase $\mathrm{C}$, Myt 1 kinase, and Tie2 kinase (16). Of these enzymes, it was determined that scytonemin acted as an inhibitor for PLK1, CDK1/cyclin B, checkpoint kinase 1, protein kinase $\mathrm{C}$, and Myt 1 kinase, but did not inhibit protein kinase A or Tie2 kinase (16). These results suggest that scytonemin binds nonspecifically to a variety of enzymes to prevent phosphorylation steps critical to cell cycle progression. Other experiments explored the relationship between scytonemin and PLK1, finding that scytonemin acts as a mixed inhibitor for PLK1 and functions in a concentration-dependent, time-independent manner to induce cell cycle arrest $(15,16$, 18). By looking at molecular signals in the cell, the specific mechanism of action of scytonemin appears to be G2 to M phase cell cycle arrest $(15,19)$.

In light of these prior findings, the results of this study support the anti-proliferative potential of scytonemin as an inhibitor of important cell cycle enzymes implicated in cancer growth. Scytonemin at $2 \mu \mathrm{M}$ and $10 \mu \mathrm{M}$ significantly inhibited melanoma cell proliferation by greater than $50 \%$ compared to controls in a somewhat concentrationdependent manner. This is consistent with other studies that explore the effect of scytonemin on malignant cells $(15,18$, 19).

In addition, the results of the spleen cell assay demonstrates that $2 \mu \mathrm{M}$ scytonemin significantly stimulated proliferation 
Figure 2.

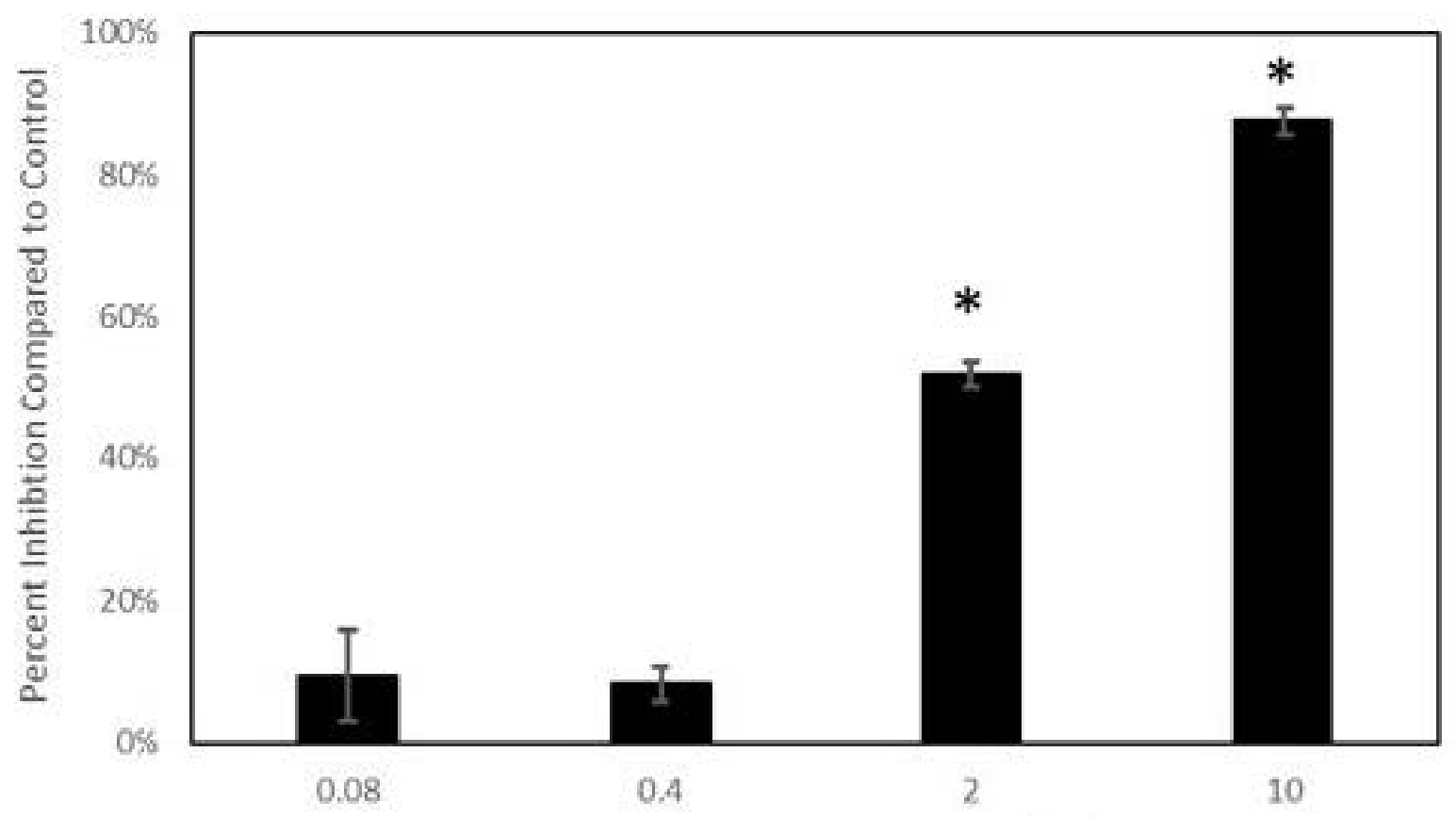

Figure 2. Percent inhibition of melanoma cells compared to the untreated control for various concentrations of scytonemin. Error bars represent the standard error of nine replicates. Significant inhibition compared to the control is marked with an asterisk, for both the $2 \mu \mathrm{M}$ and $10 \mu \mathrm{M}$ samples, $\mathrm{p}<0.0001$.

compared to the controls, which was an unexpected outcome. $\quad$ in a concentration-dependent manner through $10 \mu \mathrm{M}$ of Since scytonemin typically inhibits actively proliferating cell types (15), there was some concern that it would inhibit spleen and melanoma cell growth alike. However, this was not the case, and was especially true for spleen cells receiving Con A. In these cells scytonemin appears to have enhanced proliferation, ranging from $164.79 \%$ to $387.00 \%$ relative to the control group. However, unlike the melanoma cell assays, the spleen cells were not affected by scytonemin scytonemin. This result could be due to high standard errors or data resolution, which does not show the trend between 0.4 to $2 \mu \mathrm{M}$ of scytonemin. Nonetheless, the data suggests that scytonemin inhibits melanoma cells without hindering spleen cell proliferation. Given that scytonemin inhibits melanoma cell proliferation and potentially increases spleen cell proliferation, it is a promising therapeutic agent for melanoma treatment because it can slow cell growth while

Figure 4 .

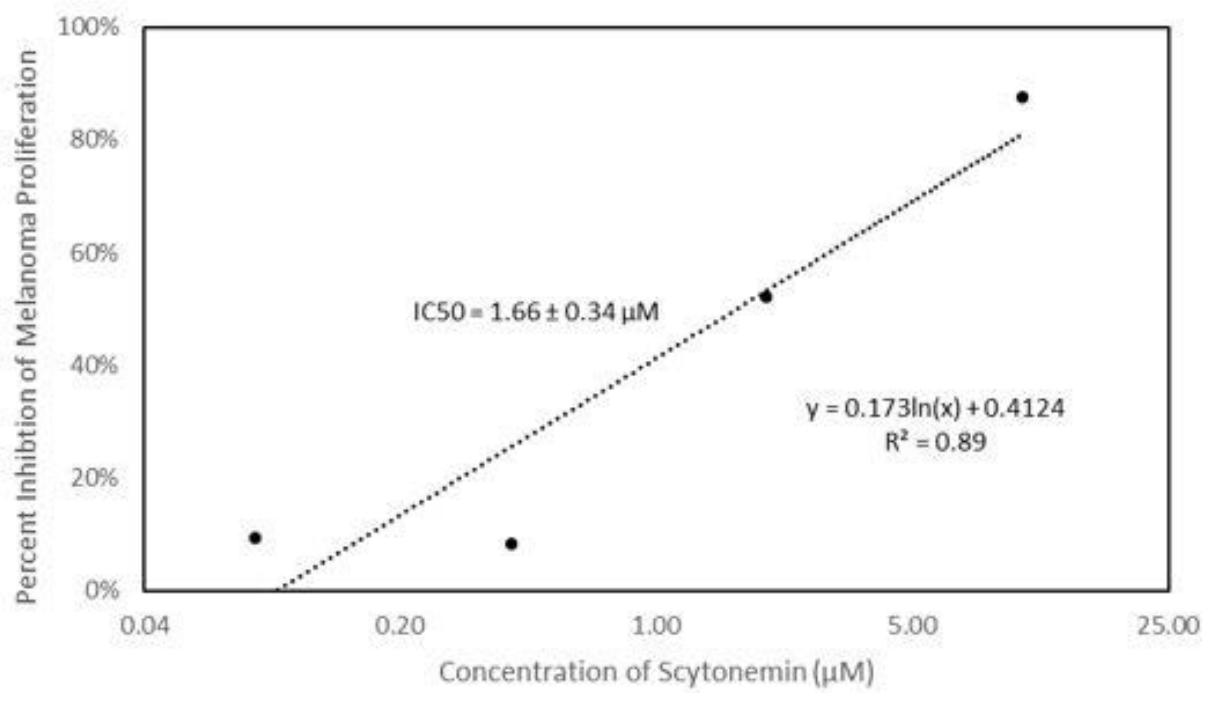


Figure 3.

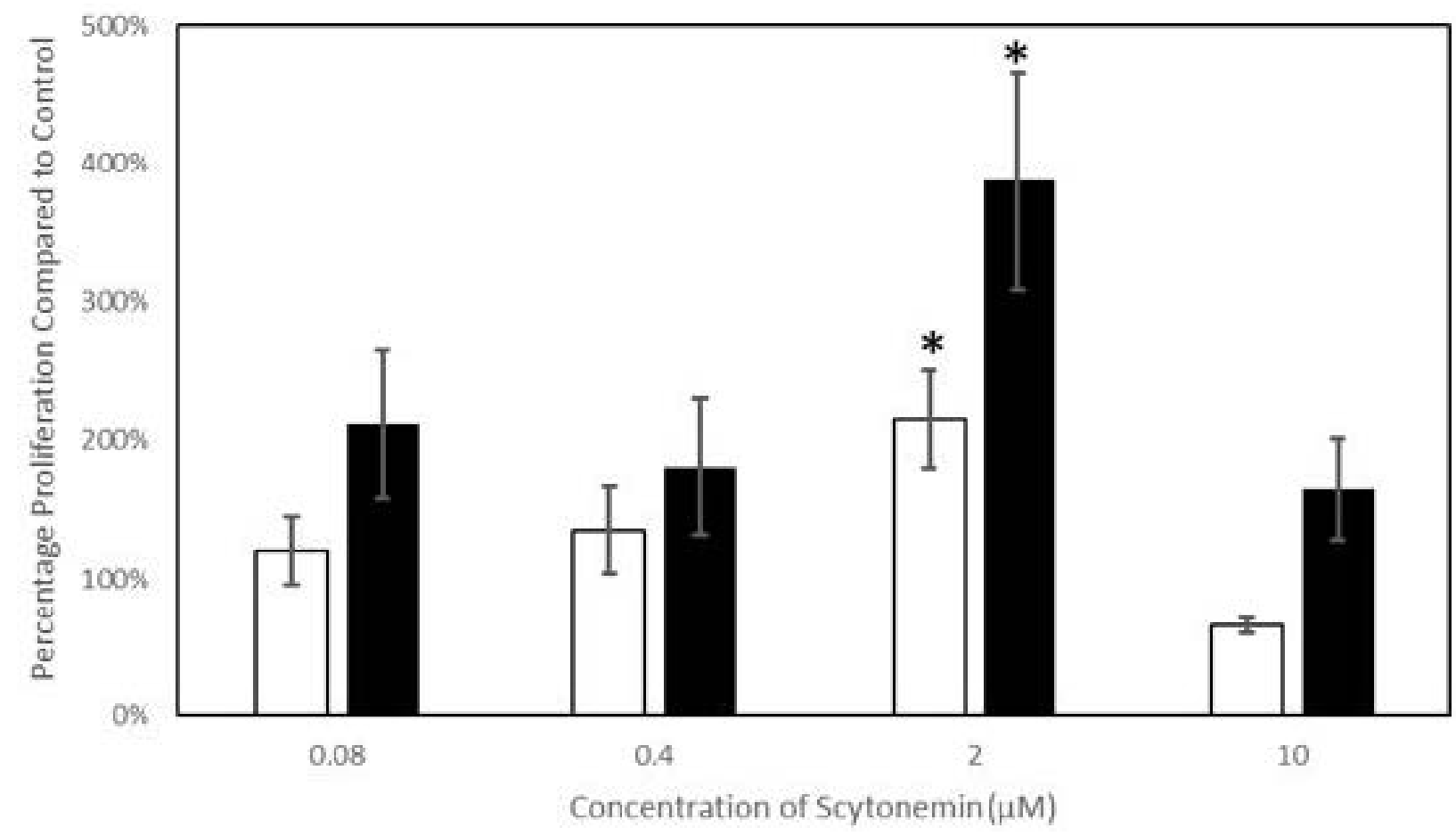

Figure 3. Percent proliferation of spleen cells compared to the control for various concentrations of scytonemin in the absence (white bars) and presence (black bars) of Con A. Error bars represent the standard error of nine replicates. Significant inhibition compared to the control is marked with an asterisk, at $2 \mu \mathrm{M}$ scytonemin, $\mathrm{p}=0.0012$ for cells with and without Con A. Biorender.com.

simultaneously augmenting the immune system. The in vivo experiments on mice evaluating the number of tumors formed with and without the presence of scytonemin favored the inhibitory effects of scytonemin. Even though there were no significant differences in the number of melanoma tumors in untreated mice versus those treated with scytonemin, the averages themselves indicate that with additional studies there may be some inhibitory effect of scytonemin on melanoma tumor cells. These studies could benefit by a dose-dependent analysis. The concentration of scytonemin used was based on a study using Jurkat $\mathrm{T}$ cells (15) and it may have not been ideal to use the same concentration in a study on melanoma tumor development.

To better compare the inhibitory effects of scytonemin with the previous body of knowledge, the inhibitory concentration at $50 \%$ inhibition (IC50) was determined. This value represents the concentration of scytonemin at which cell proliferation is stunted by $50 \%$ relative to the control. In a regression analysis of scytonemin concentration versus percent 60 | Fine Focus inhibition of melanoma proliferation, a positive correlation $(\mathrm{R} 2=0.89)$ was identified (Fig. 4). Using this regression plot, the IC50 for scytonemin on melanoma cells was determined to be approximately $1.66 \pm 0.34 \mu \mathrm{M}$. In a similar study measuring the effects of scytonemin on the cancerous Jurkat T cell line, the IC50 was determined to be $2.5 \pm 0.6 \mu \mathrm{M}$ (15). These relatively close values could help inform future research studies exploring the benefits of scytonemin as an antiproliferative therapeutic.

The results of this study support that scytonemin inhibits melanoma cell proliferation and enhances the immune response via spleen cell proliferation. As a result, future research should examine the role of scytonemin on in vivo tumor growth beyond our studies to determine whether scytonemin functionally inhibits malignant growth in body systems without cytotoxicity to healthy cells. Further, in vivo systems can be used to examine the response of the immune system to scytonemin, to potentially affirm enhanced spleen cell proliferation and examine other cytokines and immune 
cells. Research could also focus on experimental cancer therapies using scytonemin to prevent tumors from growing and metastasizing. Since a growing body of literature suggests that scytonemin acts as an inhibitor for PLK1 $(15,16,18,19)$, the role of PLK1 in melanoma and spleen cell proliferation should be further studied. In addition, since dimethoxyscytonemin binds to PLK1 with high affinity (9), it would be interesting to study how this scytonemin derivative affects proliferation in melanoma and spleen cell assays compared to purified scytonemin. Overall this study, along with prior research, provides evidence that the therapeutic potential of scytonemin should be further explored.

\section{Acknowledgements}

We wish to thank Dr. Benjamin Philmus for the purified scytonemin extract used in this study. We also thank the Purdue Fort Wayne Office of University Research and Innovation for support through the Undergraduate Summer Research Support Program. This research is in compliance with federal regulations and institutional policies relating to animal care and use according to IACUC Protocol \#1111000244.

\section{References}

1. Bebout BM, Garcia-Pichel F. 1995. UV-B-induced vertical migrations of cyanobacteria in a microbial mat. Appl Environ Microbiol 61:4215-4222.

2. Briles EB, Kornfeld S. 1978. Isolation and metastatic properties of detachment variants of B16 melanoma cells. J Natl Cancer Inst 60:1217-1222.

3. CDC. 2020. Skin Cancer. https://www.cdc.gov/cancer/skin/statistics/index.htm. Accessed

4. Ehling-Schulz M, Bilger W, Scherer S. 1997. UV-B-induced synthesis of photoprotective pigments and extracellular polysaccharides in the terrestrial cyanobacterium Nostoc commune. J Bacteriol 179:1940-1945.

5. Garcia-Pichel F, Castenholz RW. 1991. Characterization and biological implications of scytonemin, a cyanobacterial sheath pigment. J Phycol 27:395-409.

6. Garcia-Pichel F, Sherry ND, Castenholz RW. 1992. Evidence for an ultraviolet sunscreen role of the extracellular pigment scytonemin in the terrestrial cyanobacterium Chlorogloeopsis sp. Photochem Photobiol 56:17-23.

7. Huang L, McCluskey MP, Ni H, LaRossa RA. 2002. Global gene expression profiles of the cyanobacterium Synechocystis sp. strain PCC 6803 in response to irradiation with UV-B and white light. J Bacteriol 184:6845-6858.

8. Jagger J. 1985. Solar-UV actions on living cells. Praeger, New York.

9. Pathak J, Mondal S, Ahmed H, Rajneesh, Singh SP, RP S. 2019. In silico study on interaction between human polo-like kinase 1 and cyanobacterial sheath pigment scytonemin by molecular docking approach. Biointerface Res Appl Chem 9:4374-4378.

10. Proteau PJ, Gerwick WH, Garcia-Pichel F, Castenholz RW. 1993. The structure of scytonemin, an ultraviolet sunscreen pigment from the sheaths of cyanobacteria. Experientia 49:825- 829.

11. Rastogi R, Sonani R, Madamwar D. 2015. Cyanobacterial sunscreen scytonemin: Role in photoprotection and biomedical research. Appl Biochem Biotechnol 176:1551-1563. 
12. Reeke GN, Jr., Becker JW, Cunningham BA, Wang JL, Yahara I, Edelman GM. 1975. Structure and function of concanavalin A. Adv Exp Med Biol 55:13-33.

13. RStudio. 2018. RStudio: Integrated Development for R. RStudio, Inc., Boston, MA. http:// www.rstudio.com/.

14. Shibata H, Baba K, Ochiai H. 1991. Near-UV induces shock proteins in Anacystis nidulans R-2, possible role of active oxygen. Plant Cell Physiol 32:771-776.

15. Stevenson CS, Capper EA, Roshak AK, Marquez B, Eichman C, Jackson JR, Mattern M, Gerwick WH, Jacobs RS, Marshall LA. 2002. The identification and characterization of the marine natural product scytonemin as a novel antiproliferative pharmacophore. J Pharmacol Exp Ther 303:858-866.

16. Stevenson CS, Capper EA, Roshak AK, Marquez B, Grace K, Gerwick WH, Jacobs RS, Marshall LA. 2002. Scytonemin, a marine natural product inhibitor of kinases key in hyperproliferative inflammatory diseases. Inflammation Res 51:112114.

17. Urbach F. 1997. Ultraviolet radiation and skin cancer of humans. J Photochem Photobiol B: Biology 40:3-7.

18. Zhang G, Zhang Z, Liu Z. 2013. Polo-like kinase 1 is overexpressed in renal cancer and participates in the proliferation and invasion of renal cancer cells. Tumor Biol 34:1887-1894.

19. Zhang G, Zhang Z, Liu Z. 2013. Scytonemin inhibits cell proliferation and arrests cell cycle through downregulating Plk1 activity in multiple myeloma cells. Tumor Biol 34:2241-2247. 
Volume Seven | 63 